\title{
Multiple orbital neurofibromas, painful peripheral nerve tumors, distinctive face and marfanoid habitus: a new syndrome
}

\author{
D Babovic-Vuksanovic ${ }^{\star, 1}$, Ludwine Messiaen ${ }^{2}$, Christoph $\mathrm{Nagel}^{3}$, Hilde Brems ${ }^{4}$, Bernd Scheithauer ${ }^{5}$, \\ Ellen Denayer ${ }^{4}$, Rong $\mathrm{Mao}^{6}$, Raf Sciot ${ }^{7}$, Karen M Janowski ${ }^{2}$, Martin U Schuhmann ${ }^{3}$, Kathleen Claes ${ }^{8}$, \\ Eline Beert ${ }^{4}$, James A Garrity ${ }^{9}$, Robert J Spinner ${ }^{10}$, Anat Stemmer-Rachamimov ${ }^{11}$, Ralitza Gavrilova ${ }^{1}$, \\ Frank Van Calenbergh ${ }^{12}$, Victor Mautner ${ }^{13}$ and Eric Legius ${ }^{4}$
}

Four unrelated patients having an unusual clinical phenotype, including multiple peripheral nerve sheath tumors, are reported. Their clinical features were not typical of any known familial tumor syndrome. The patients had multiple painful neurofibromas, including bilateral orbital plexiform neurofibromas, and spinal as well as mucosal neurofibromas. In addition, they exhibited a marfanoid habitus, shared similar facial features, and had enlarged corneal nerves as well as neuronal migration defects.

Comprehensive NF1, NF2 and SMARCB1 mutation analyses revealed no mutation in blood lymphocytes and in schwann cells cultured from plexiform neurofibromas. Furthermore, no mutations in RET, PRKAR1A, PTEN and other RAS-pathway genes were found in blood leukocytes. Collectively, the clinical and pathological findings in these four cases fit no known syndrome and likely represent a new disorder.

European Journal of Human Genetics (2012) 20, 618-625; doi:10.1038/ejhg.2011.275; published online 18 January 2012

Keywords: orbital neurofibroma; plexiform neurofibroma; enlarged corneal nerves; marfanoid habitus

\section{INTRODUCTION}

Peripheral nerve sheath tumors, specifically neurofibromas and schwannoma variants, occur both sporadically and in the setting of hereditary conditions, including neurofibromatosis type 1 (NF1), neurofibromatosis type 2 (NF2), and familial/sporadic schwannomatosis. The diagnosis of these disorders is usually based upon clinical criteria, but can be confirmed by DNA testing. In rare instances, confirming the diagnosis is difficult because of an attenuated clinical presentation resulting from tissue mosaicism for the specific mutation. ${ }^{1}$ Neurogenic tumors are also observed in other, more rare conditions. For example, Carney complex, an autosomal dominant, multiple neoplasia syndrome, is characterized by cardiac and cutaneous myxomas, various pigmented lesions of skin and mucosae, endocrine processes affecting the adrenal and pituitary glands, as well as psammomatous melanotic schwannomas. ${ }^{2}$ Neuromas of the eyelids, lip and tongue as well as visceral ganglioneuromatosis and enlarged corneal nerves are observed in multiple endocrine neoplasia type 2B (MEN2B), an autosomal dominant condition also characterized by distinctive facies, a marfanoid habitus and medullary thyroid carcinoma (MTC). ${ }^{3}$ Multiple mucosal and cutaneous neuromas, as well as prominent corneal nerves, may be seen in PTEN hamartoma tumor syndrome (PHTS). ${ }^{4}$ In addition, patients with Bannayan-Riley-
Ruvalcaba syndrome, a part of the PHTS spectrum, develop caféau-lait spots and macules of the glans penis.

Herein, we present four unrelated patients with strikingly similar clinical features, including bilateral orbital and multiple systemic, mucin-rich plexiform neurofibromas, enlarged corneal nerves, and a marfanoid habitus. This spectrum of clinical features differs from that of any known condition and likely represents a new, previously unrecognized syndrome.

\section{MATERIALS AND METHODS, AND RESULTS \\ Pathology \\ The specimens were fixed in 10\% buffered formalin, routinely processed and paraffin-embedded. Five micron sections were stained by the hematoxylin-eosin as well as Alcian blue methods, and by immunohistochemistry (streptavidin-biotin peroxidase complex method) for S-100 protein (Dako, Carpinteria, CA, USA; polyclonal; 1:400), epithelial membrane antigen (EMA) (Dako; clone 9867; 1:50) and neurofilament protein (Dako; clone 2F11; 1:50). All tumors represented neurofibromas, most of plexiform type. Plexiform lesions showed EMA-positive perineurium expanded by variably cellular endoneurium containing Alcian blue-positive mucoid matrix. Endoneurial infiltrates of schwann cells were cytologically benign}

\footnotetext{
${ }^{1}$ Department of Medical Genetics, Mayo College of Medicine, Rochester, MN, USA; ${ }^{2}$ Department of Genetics, University of Alabama at Birmingham, Birmingham, AL, USA; ${ }^{3}$ Department of Neurosurgery, University of Tuebingen, Tubingen, Germany; ${ }^{4}$ Department of Human Genetics, University Hospital Leuven, Leuven, Belgium; ${ }^{5}$ Department of Laboratory Medicine \& Pathology, Mayo College of Medicine, Rochester, MN, USA; ${ }^{6}$ Molecular Genetics Section, ARUP Laboratories, Salt Lake City, UT, USA; ${ }^{7}$ Department of Pathology, University Hospital Leuven, Leuven, Belgium; ${ }^{8}$ Department of Medical Genetics, University of Gent, Gent, Belgium; 9 Department of Ophthalmology, Orbital Surgery \& Neuro-ophthalmology, Mayo College of Medicine, Rochester, MN, USA; ${ }^{10}$ Department of Neurosurgery, Mayo College of Medicine, Rochester, MN, USA; ${ }^{11}$ Department of Pathology, Harvard Medical School, Boston, MA, USA; ${ }^{12}$ Department of Neurosurgery, University Hospital Leuven, Leuven, Belgium; ${ }^{13}$ Department of Neurology, University Hospital Hamburg-Eppendorf, Hamburg, Germany

*Correspondence: Dr D Babovic-Vuksanovic, Department of Medical Genetics, Mayo Clinic College of Medicine, 200 First Street SW, Rochester, MN 55905, USA. Tel: +1 507266 2967; Fax: +1 507284 1067; E-mail: dbabovic@mayo.edu
}

Received 12 September 2011; revised 20 December 2011; accepted 22 December 2011; published online 18 January 2012 
and strongly S-100 protein positive. Axons lay variably distributed but were often centrally grouped within expanded fascicles. No clinically significant atypia was evident in any case.

\section{Case reports}

Patient 1. The patient presented at 13 years of age with progressive headache and bilateral swelling of the upper eyelids. Ophthalmological examination revealed orbital soft tissue swelling with bilateral ptosis and proptosis (Figure 1a), in addition to thickened corneal nerves (Figure 1b). A magnetic resonance imaging (MRI) scan disclosed bilateral orbital tumors, massive tumoral involvement of the trigeminal and vidian nerves, as well as the presence of multiple small neurogenic tumors in subcutaneous tissues of the head and neck, mainly in a facial nerve distribution (Figures $1 \mathrm{c}$ and d). An incidental finding was a right frontal lobe neuronal migration anomaly consisting of closed left schizencephaly extending to the right anterior frontal horn and polymicrogyria (Figure 1e). No radiological evidence of optic glioma, vestibular schwannoma, meningiomas, ependymomas, or of the cerebral T2 hyperintensities commonly associated with NF1 was noted.

The patient's past medical and family history was unremarkable. Aside from an elevated maternal blood alpha-fetoprotein level, she was born after a normal term pregnancy (birth weight $4500 \mathrm{~g}$ ). Cytogenetic analysis of amniocytes demonstrated a normal karyotype (46,XX). An umbilical hernia was present at birth, but had closed spontaneously. Psychomotor development had been normal. Physical examination disclosed a marfanoid habitus and somewhat coarse facial features. Her height was $173 \mathrm{~cm}(>97 \%)$ at a weight of $65 \mathrm{~kg}$ (91\%), body mass index (BMI) $22.5 \mathrm{~kg} / \mathrm{m}^{2}$. The arm-span:height ratio was increased at 1.06 and her head circumference was $55 \mathrm{~cm}$ (90-95\%). In addition to ocular proptosis, unusual facial features included nasal prominence, full thick lips, and several submucosal protuberances in both lips and the sublingual zone (Figure 1a). Several small, firm, painful and well-delineated subcutaneous nodules of head and face were noted, particularly in the pre- and postauricular areas of the scalp and in the neck. The patient had a mild pectus excavatum chest deformity but not scoliosis. Five hyperpigmented, irregularly bordered $2-3 \mathrm{~cm}$ skin macules were noted, but no mucosal or intertriginous freckling was seen. The scars after surgery were hyperplastic with keloid appearance. Affecting the frontal nerves, the orbital tumors (Figures if and g) were partly resected on two occasions. Histologically, the resected lesion consisted of WHO (World Health Organization) grade I plexiform neurofibromas (Figures $2 \mathrm{a}-\mathrm{c}$ ); mucin was focally abundant. Biopsy of one of the sublingual tumors revealed similar features with multiple-affected fascicles (Figure 2d). The patient underwent resection of multiple painful neurofibromas from the area of head and neck, including lower lip, which revealed same histological findings. Spine MRI at age 15 years was normal, but imaging 2 years later showed multiple small neurofibromas affecting sacral nerve roots.

A comprehensive analysis for NF1 gene mutations was conducted in the Medical Genomics Laboratory of the University of Alabama, Birmingham. No mutation was detected in a blood sample as well as in schwann cells cultured from the orbital tumor. Sequence analysis of all exons of the PTPN11, RAF1, SOS1, KRAS, NRAS, CBL, SHOC2, and SMARCB1 genes (Medical Genomics Laboratory of the University of Alabama, Birmingham) did not reveal any mutation. As the possibility of MEN2B was raised, the patient underwent a detailed endocrine workup, which showed no evidence of hormone-secreting tumors. RET gene sequencing was negative aside from the presence of a maternally inherited heterozygous c.2071G > A, p.Gly691Ser polymorphism (ARUP Laboratories, Salt Lake City, UT, USA). Carney complex also was considered. A thyroid ultrasound disclosed a tiny
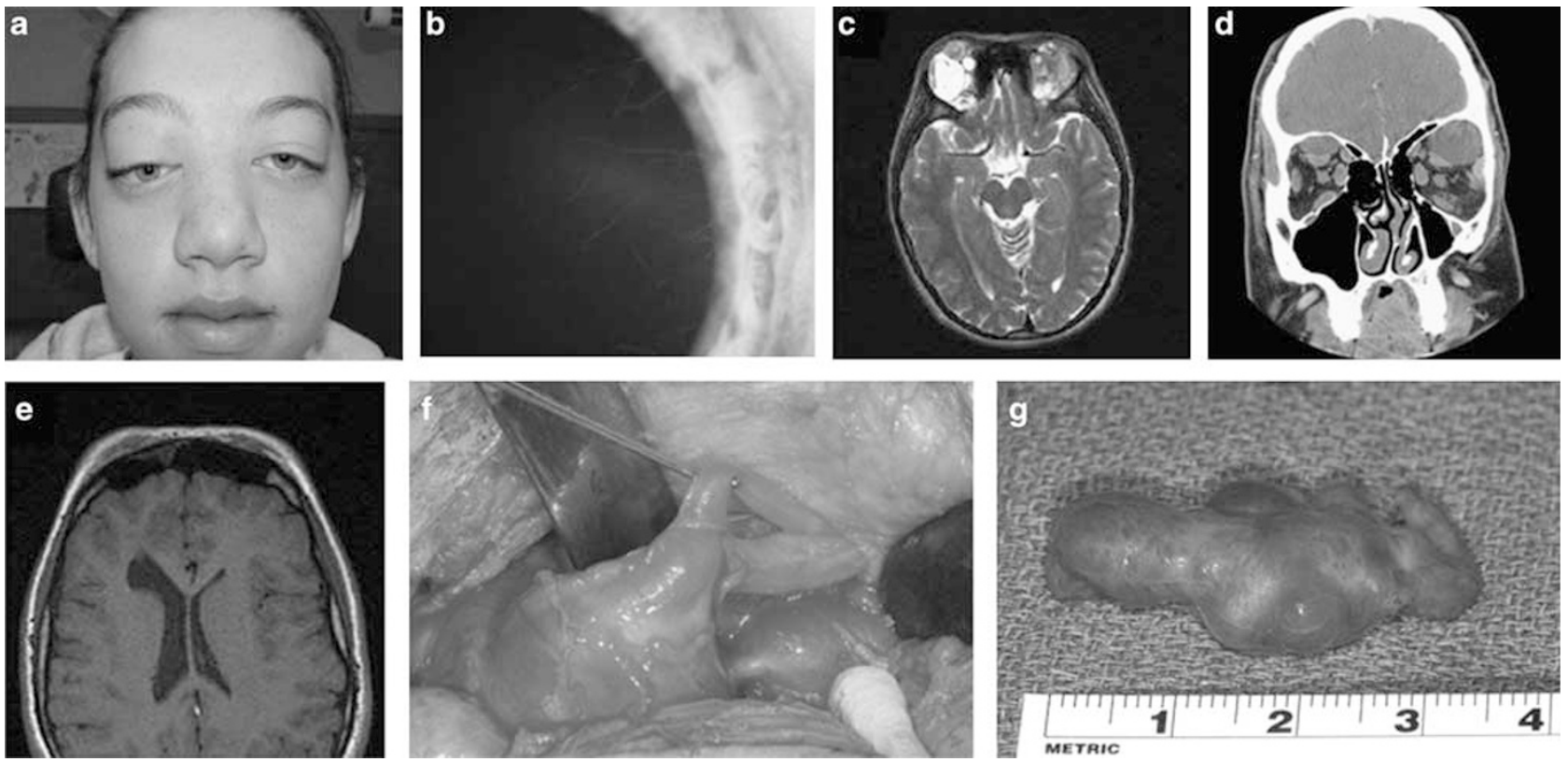

Figure 1 Patient 1. Facial features with mild dysmorphism and facial freckling. Ptosis and eyelids swelling due to bilateral plexiform neurofibromas, coarse facial features, and full lips (a). Thickened corneal nerves (b), MRI brain (c) and computed tomography of orbits (d). Numerous enhancing mass lesions consistent with nerve sheath tumors within the orbits bilaterally (e). Enhancing mass lesions in the anterior parotid glands bilaterally, of the divisions of the fifth cranial nerves as well as the vidian nerves (d). Changes of a migrational anomaly involving the right anterior frontal lobe. This configuration suggests polymicrogyria in association with closed right schizencephaly extending to the right anterior frontal horn of the lateral ventricle (e). Massive enlargement of orbital nerves and multiple orbital tumors intraoperative view (f). Removed plexiform neurofibroma involving the branch of frontal nerve (g). 

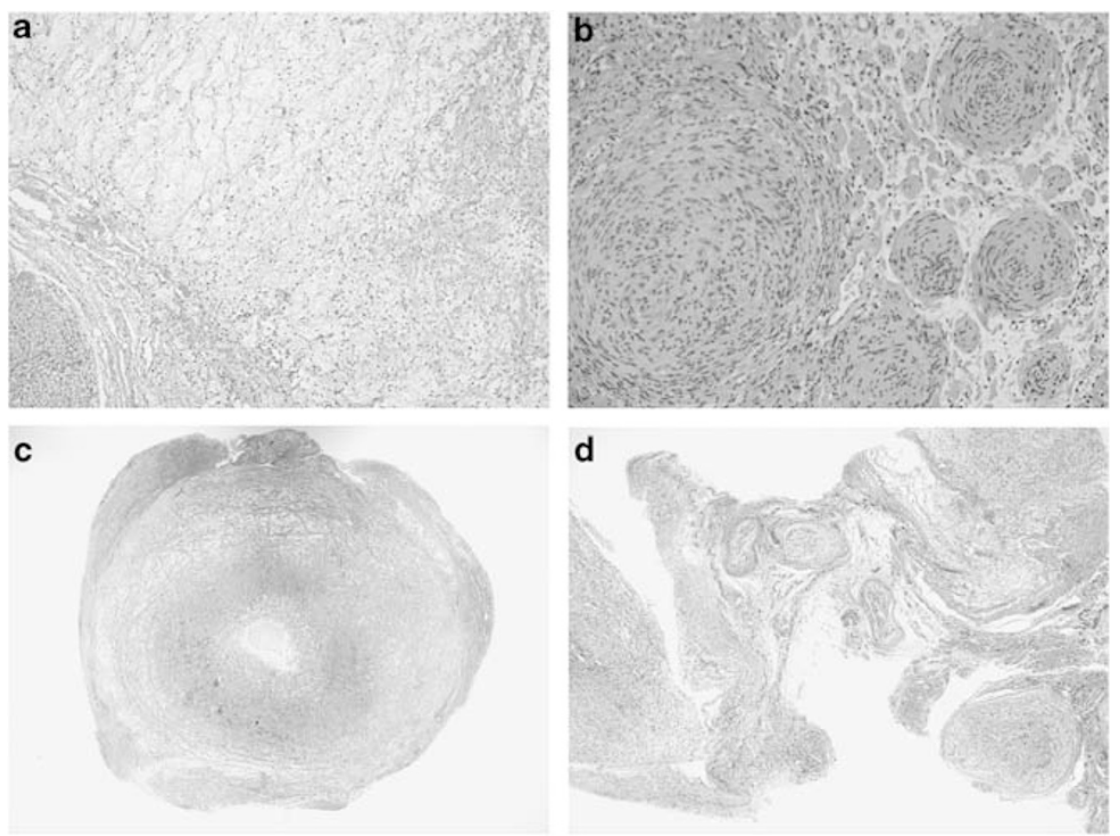

Figure 2 Patient 1. Plexiform neurofibromas. The right orbital lesion showed some fascicles to be mucin-rich (a). In addition, whorls of schwann cells were a focal conspicuous finding (b). Cross section of one fascicle showed the characteristic, centrally situated residual nerve surrounded by tumor tissue (c). Sublingual tumor (d) revealed similar features; note multiple affected fascicles.

nodule suggestive of lymphocytic thyroiditis. An echocardiogram revealed an atrial septal aneurysm without a shunt, but no evidence of atrial myxoma. Sequencing of exons 2-11 of the PRKAR1A gene (GeneDx Laboratory, Gaithersburg, MD, USA) revealed no mutations. In addition, analysis of exons 1-9 of the PTEN gene showed no alteration. An array comparative genomic hybridization (CGH) assay using a custom oligonucleotide microarray (Agilent Technologies Inc., Santa Clara, CA, USA) showed duplication of 14 nucleotide probes at 17q12 spanning approximately 925 kilobases; parental studies revealed the same chromosomal finding in the patient's unaffected mother.

Patient 2. Having a complex medical history, this patient with left hemiparesis first came to our attention at the age 15 years. She had epilepsy and learning difficulties requiring special education. Right leg muscle atrophy was attributed to 'neurofibromatous neuropathy'. Multiple small, painful subcutaneous peripheral nerve tumors had been operated upon multiple occasions. She had also undergone multiple surgeries for Carpal Tunnel syndrome. Given her marfanoid habitus, coarse facial features, full lips, gingival hyperplasia, and enlarged corneal nerves, the patient had long been followed for presumed MEN2B (Figure 3a). Clinical examination showed no pigmentary abnormalities (café-au-lait spots, intertriginous freckling) suggestive for NF1; only multiple facial freckles were present. The patient had mucosal neurofibromas (Figure 3b). An MRI showed an irregular configuration of right frontal gyri with associated closed schizencephaly and dilation of the frontal horn of the adjacent lateral ventricle (Figure 3c). A spinal MRI scan showed paraspinal nerve root masses involving almost all spinal levels, consistent with peripheral nerve sheath tumors. The thoracic spine had the largest tumor burden (Figure 3d). There was also diffuse enlargement of the brachial plexus, lumbosacral plexus, and sciatic nerves (Figures $3 e$ and $\mathrm{f}$ ). Ultrasonography and MRI scans of the neck showed no thyroid abnormality.

In ensuing years, the patient underwent surgery for removal of several small subcutaneous nodules involving the left foot, left hip, right pelvis, right knee and multiple nodules affecting the right median nerve. Each had presented as a palpable, painful mass.

At age 22, the patient presented with left monocular diplopia. Her height was $165 \mathrm{~cm}$ at a weight of $44 \mathrm{~kg}$ and a BMI of $16.1 \mathrm{~kg} / \mathrm{m}^{2}$. An MRI scan revealed multiple tumors in the right orbit, presumably neurofibromas (Figure $3 \mathrm{~g}$ ). Bilateral enlargement of the maxillary and infraorbital nerves was also noted. At 6 months thereafter, she presented with a polypoid submucosal swelling in the right nostril (Figure 3h). The lesion was resected; histology revealed a somewhat atypical WHO grade I neurofibroma (Figure 4). Mucin was abundant. Mutation analyses of blood leukocytes and of cultured schwann cells from the tumor showed no mutations in the NF1, NF2, RET and SMARCB1 genes. A RET mutation analysis was also normal in DNA extracted from neurofibroma tissue (not cultured). PTEN and PRKAR1A mutation analyses of blood leukocytes were negative. Routine chromosome analysis and CGH (using Agilent 244K arrays) of peripheral blood, cultured schwann cells, and fibroblasts of the nasal tumor showed no abnormalities (data not shown).

Patient 3. The patient, a 24-year-old woman, came to the outpatient clinic with bilateral lumbosacral pain, right foot weakness and numbness. Her medical history included excisions of subcutaneous nodules from the popliteal fossa 6 years before and removal of nodules from both lips at age 20. Owing to retrognathia, a maxillary osteotomy had been performed. She was blind in the left eye because of juvenile glaucoma for which she had undergone a trabeculotomy at age 18 . A marfanoid habitus and severe scoliosis had been apparent since puberty. At age 17, estrogen had been administered to halt linear growth.

Clinical examination disclosed a body height of $189 \mathrm{~cm}$ at a weight of $58 \mathrm{~kg}$ and BMI of $16.2 \mathrm{~kg} / \mathrm{m}^{2}$. Her joints were hypermobile. Arachnodactyly, pectus excavatum and multiple painful subcutaneous nodules of the neck and trunk were noted. No café-au-lait spots, axillary or inguinal freckling, or Lisch nodules were seen to suggest NF1. She had bilateral proptosis. Neurological examination revealed 

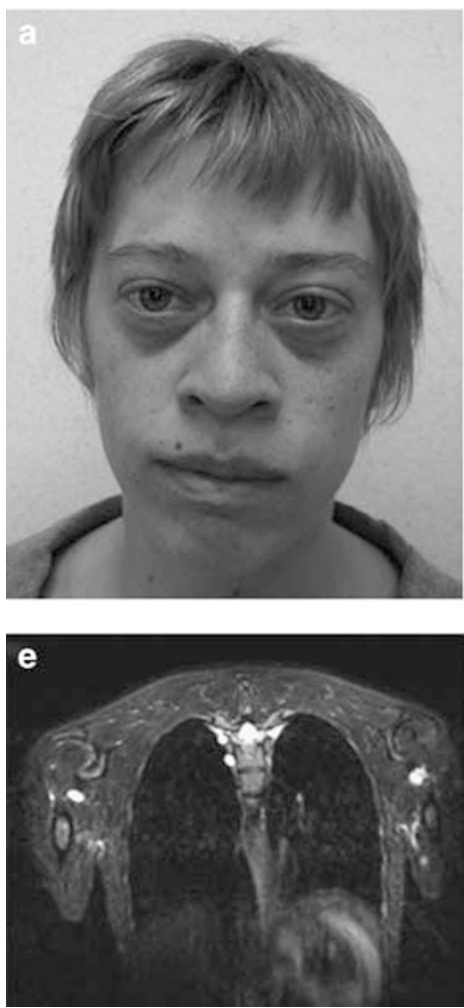
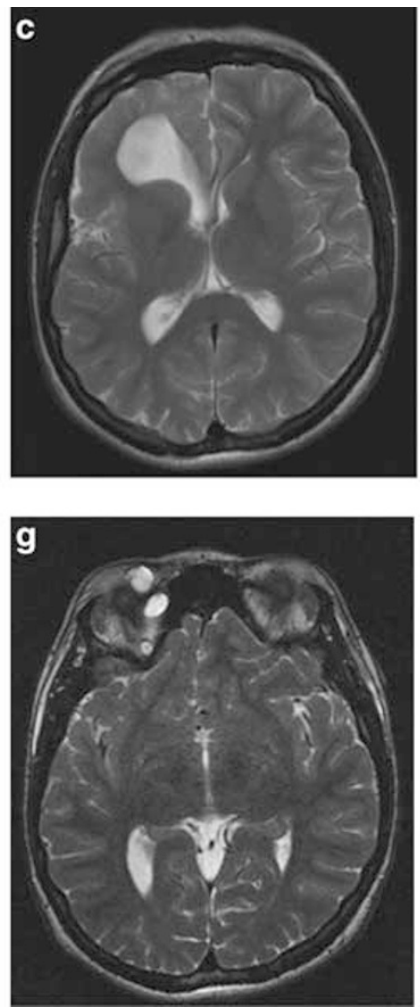

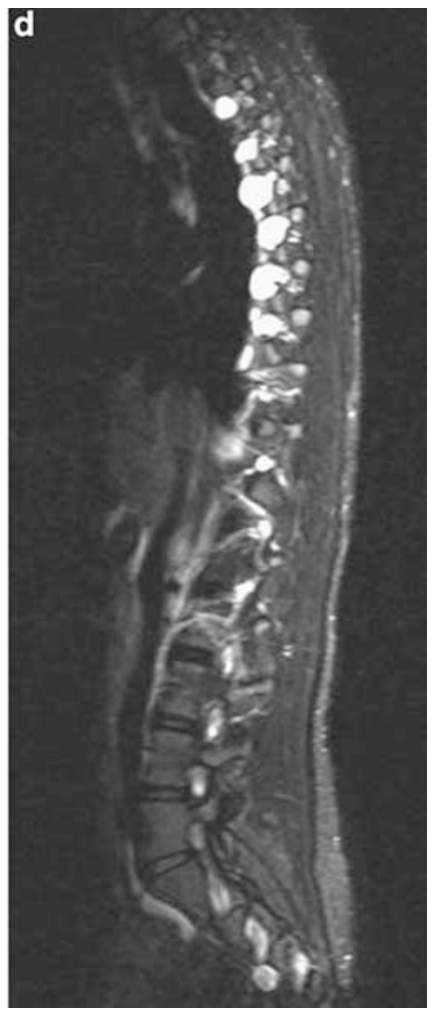

Figure 3 Patient 2. Proptosis due to bilateral orbital tumors, full lips (a), and mucosal neurofibromas (b). MRI of the brain shows migrational anomaly involving the right anterior frontal lobe. The configuration suggests polymicrogyria in association with closed right schizencephaly extending to the right anterior frontal horn of the lateral ventricle (c). MRI spine shows numerous enhancing mass lesions extending through the neural foramina at multiple spinal levels, most prominent at the lower cervical and upper thoracic levels, consistent with nerve sheath tumors (d). Involvement of bilateral brachial (e) and lumbosacral (f) plexi with multiple neurofibromas. MRI of the orbits shows two enhancing mass lesions consistent with nerve sheath tumors within the right orbit (g). Polypoid mass in the right nostril (h).

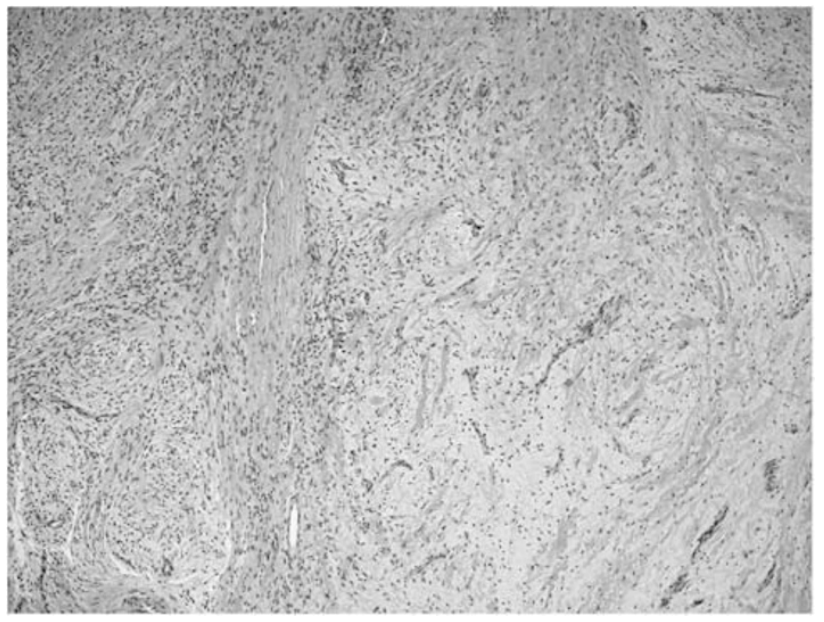

Figure 4 Patient 2. Neurofibroma, right nostril. Note mild clinically insignificant atypia consisting of mild hypercellularity and hyperchromasia.

blindness on the left eye, paresis of the right foot, hypoesthesia of both feet, decreased (3/8) vibrational sensation at the ankles, and moderate ataxia. Nerve conduction studies revealed severe axonal and demyelinating sensorimotor polyneuropathy. Electromyography showed denervation of the right tibialis anterior muscle. MRI scans of the head and spine showed multiple neurofibromas, affecting orbits and cavernous sinuses (Figures $5 \mathrm{a}-\mathrm{c}$ ), multiple spinal nerve roots
(Figure 5d), and both brachial as well as lumbosacral plexuses (Figure 5e). The brain and spinal cord appeared normal.

Six months later, a left orbital tumor was resected. Histologically, it was a WHO grade I plexiform neurofibroma (Figure 6a). Mucin was again abundant (Figures $6 \mathrm{~b}$ and c). Further technical and laboratory studies performed at that time were described in detail by Hartlapp et al. ${ }^{5}$ In addition to multiple neurogenic tumors and the marfanoid habitus, she had prominent scoliosis as well as bilateral acetabular protrusions, mitral valve prolapse, and a dilated ascending aorta.

At age 30, the patient returned with complaints of severe, progressive pain at several locations. This was attributed to multiple solid tumors along the course of peripheral nerves mainly affecting her right arm, neck, inguinal areas and thighs. She underwent a series of surgical procedures for resection of a very painful tumor of the right ulnar nerve proximal to the elbow. Large tumors of both femoral nerves required microsurgery. The tumors were classified as WHO grade I neurofibromas. Their morphology differed somewhat in that both featured prominent schwannian nodules as seen in case 1. After the last procedure, the patient entered a rehabilitation facility where, one morning, she was found dead in bed. Her cause of death remains unexplained but could be related to known dilation of the ascending aorta, previously documented on echocardiography. No autopsy was performed. NF1 mutation analysis in blood DNA was negative and HPLC (high performance liquid chromatography) of tumor material (schwann cells) did not reveal a mutation in the NF1 gene.

Patient 4. At age 18, the patient presented with a large orbital plexiform neurofibroma and consequent glaucoma of the left eye 

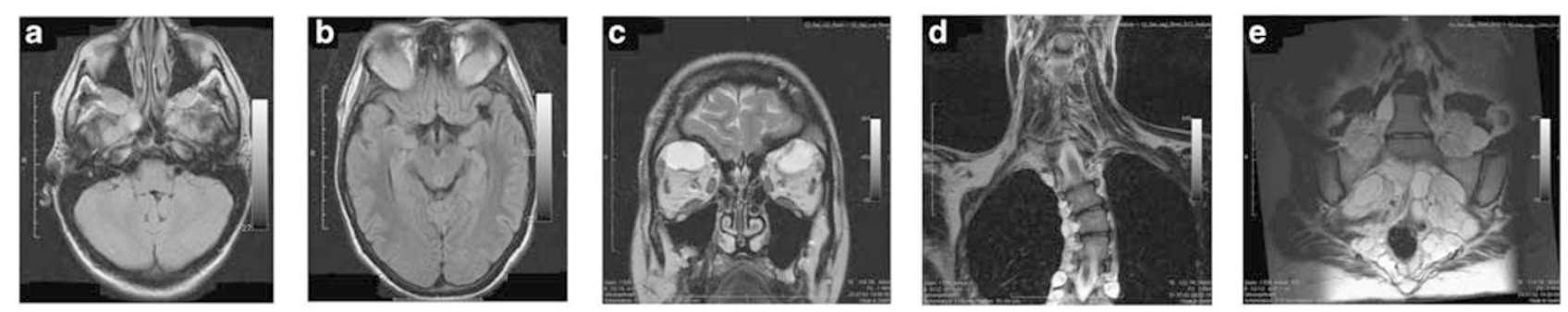

Figure 5 Patient 3. Limited MRI imaging was available. MRI brain with mass lesions at the skull base (a). Subcutaneous neurofibroma in the right temporal area (b). Multiple orbital neurofibromas bilaterally (c). Neurofibromas involving multiple thoracic nerve roots (d) and massive bundle of pelvic tumors (note thoracic scoliosis as well) (e).
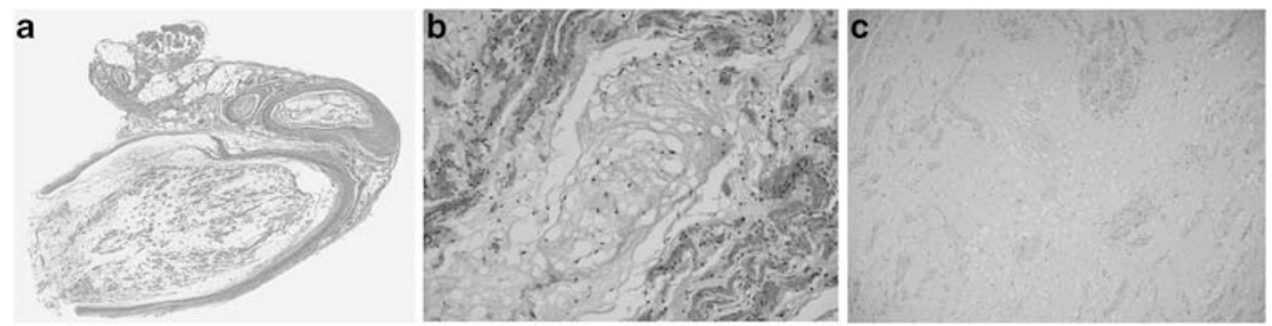

Figure 6 Patient 3. Plexiform neurofibroma, left orbit. Note varying expansion of three fascicles (a) and relative abundance of mucin (b), showing strong staining for Alcian blue (c).
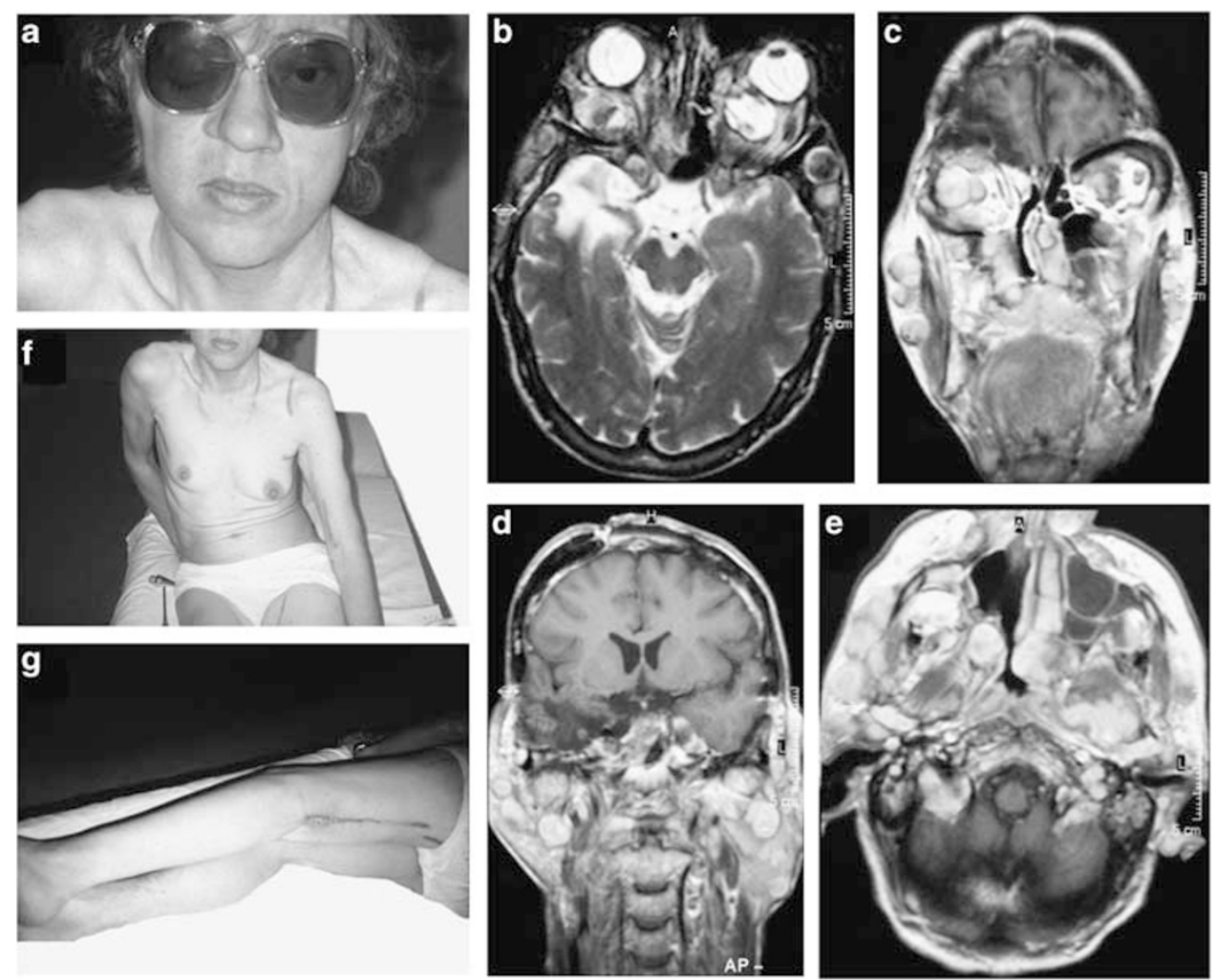

Figure 7 Patient 4. Asymmetric proptosis due to bilateral orbital tumors and full lips (a). MRI brain and orbits show innumerable neurogenic tumors including bilateral orbital neurofibromas (b-e). Numerous bilateral enhancing mass lesions in the orbits, parotid glands, superficial and deep upper face, masticator region and skull base (limited imaging was available). Marfanoid habitus and multiple keloid scars (f, $\mathbf{g}$ ).

(Figure 7a). In addition to significant proptosis and gradual loss of vision, extension of the tumor to the middle cranial fossa resulted in brain stem compression, which required neurosurgical intervention.

Starting at age 25 , the patient developed multiple painful subcutaneous neurofibromas and underwent multiple surgeries for tumor removal. Those tumors were primarily located in the left thigh, both orbits and in the skull base region.

At age 30, she underwent another surgery for excision of neurofibroma from the left sciatic nerve owing to increasing pain and neurological dysfunction of the left lower extremity. Painful 
subcutaneous tumors of the left upper arm and left axilla were also removed. In addition to multiple surgical treatments, the patient required continuous use of narcotics for control of debilitating pain.

Significant clinical deterioration due to rapid growth of multiple tumors was noted at age 40. In addition to enlargement of bilateral orbital tumors and the neurofibroma extending into the middle cranial fossa (Figures 7b-e), multiple tumors developed in the area of face, particularly in the maxillary and retro-maxillary regions. In the following years her medical condition progressively worsened, resulting in almost complete loss of vision, paralysis of the left leg, and development of bladder dysfunction. She became wheelchair-bound and required intensive pain management.

At age 59, the patient developed deep venous thrombosis and died from pneumonia and right heart failure.

At age 45, the patient's height was $162 \mathrm{~cm}$ and weight was $52 \mathrm{~kg}$ (BMI $20.3 \mathrm{~kg} / \mathrm{m}^{2}$ ). She had marfanoid habitus (Figure $7 \mathrm{f}$ ), asymmetric proptosis and full lips (Figure 7a). On clinical examination there were several firm, painful, subcutaneous neurofibromas, particularly numerous at the left arm and left leg. There were prominent hypertrophic, keloid scars in the areas of tumor removal (Figures $7 \mathrm{f}$ and g). No café-au-lait spots, Lisch nodules, axillary or inguinal freckling were present. The vision was significantly decreased on the left and absent on the right eye. The patient had anosmia, trigeminal nerve dysfunction, muscular atrophy in all extremities (more prominent on the left side), and paralysis of the left leg.

Autopsy revealed the presence of numerous tumors in diffuse distribution. Histology of lesions was consistent with plexiform neurofibromas, which had various degrees of collagen and mucoid matrix. NF1 and NF2 mutation analysis in blood DNA was negative. HPLC of tumor material did not reveal a mutation in the NF1 gene.

\section{DISCUSSION}

Peripheral nerve tumors are hallmarks of several well-known familial tumor syndromes, including NF1 and NF2, familial and sporadic schwannomatosis, and MEN2B. Isolated neurofibromas and schwannomas are also encountered in other rare, heritable conditions, such as Carney complex and PHTS. They, of course, also occur sporadically in otherwise healthy individuals.

Herein, we presented four unrelated patients with a strikingly similar clinical phenotype, which included multiple painful neurofibromas of mucosal, cranial and large spinal nerves, bilateral and multiple orbital neurofibromas, enlarged corneal nerves, congenital neuronal migration abnormalities, as well as distinctive facial features and a marfanoid habitus. Clinical findings in our four cases and one similar case from the literature (Meyer and $\mathrm{Wobig}^{26}$ ) are presented in Table 1 . We have included that patient despite the fact that testing in this case was less complete. Except for facial freckling, no other pigmentary skin changes were present; specifically there were no café-au lait spots, intertriginous freckling or Lisch nodules. No cutaneous neurofibromas were present. Unlike NF1, subcutaneous tumors were remarkably painful and had a tendency to rapidly enlarge. The pain associated with the neurofibromas in our patients is intriguing, as pain is not usually observed in the context of sporadic or NF1-associated plexiform neurofibromas. Histological examination of the tumors did not reveal any pathology that would readily explain the pain such as inflammation, thrombosis or acute hemorrhage. The tumors, however, are remarkable in that some of the histological features, specifically the abundance of mucin (myxoid stroma) and prominent schwann cell nodules resemble variants of neurofibromas or lesions with hybrid (schwannoma/neurofibroma) features. Although prominent myxoid change and tumors with hybrid histological features have been associated with schwannomatosis (and therefore often associated with pain), the tumors in schwannomatosis are schwannomas (not neurofibromas), and there is no direct evidence or functional data that links myxoid stroma or hybrid histological features with pain.

Thus, the etiology of pain in our patients remains unknown but possibility of pro-inflammatory cytokines or nerve growth factor as a major mediator of painful response can not be excluded. ${ }^{6,7}$

All cases appeared to be sporadic in occurrence as there was no reported family history of similar features. Interestingly, all four patients are female, which may be coincidental but could also represent the gender bias in disease expression. Mutational analysis of peripheral blood leukocytes showed no pathogenic mutation in the NF1, NF2, SMARCB1, PTEN, PRKAR1A, PTPN11, RAF1, SOS1, KRAS, NRAS, CBL, and SHOC2 genes. In addition, mutational analysis of tumor-derived schwann cells revealed normal NF1, NF2, and SMARCB1 genes, thus excluding mosaicism for these gene mutations. We also excluded a small deletion or duplication as the cause of the condition by performing array CGH upon blood and tumor tissue.

Although our patients shared some clinical features with MEN2B, particularly mucosal neuromas, full lips, thickened corneal nerves, and a marfanoid habitus, there were deviations from the MEN2 phenotype. They included the presence of bilateral orbital neurofibromas, multiple peripheral nerve tumors, neuropathy and developmental brain abnormalities, features unassociated with MEN2B. In addition, no RET gene mutation was observed in our probands. In patient 1 , analysis of the RET gene was negative, aside from the presence of a heterozygote p.Gly691Ser polymorphism known to be overrepresented in MTC and a possible modifier of MEN2A expression. ${ }^{8,9}$ The same polymorphism was detected in peripheral leukocytes in the patient's unaffected mother. The p.Gly691Ser polymorphism in our patient was likely a familial variant of doubtful clinical significance. In patient 2 , the RET mutation analysis was normal in cultured neurofibromaderived schwann cells and fibroblasts, as well as in DNA extracted from uncultured neurofibroma tissue. This excluded mosaicism for a $R E T$ mutation as an explanation of the atypical clinical findings. Analysis of RET gene was not performed in patients 3 and 4; the information for patients 3 and 4 was obtained retrospectively (post mortem), and therefore was somewhat limited. However, similarities in phenotypes and lack of mutations in NF1 gene in all four cases described here suggest that they all have represented the same clinical entity.

There have been several reports in the literature of patients with atypical MEN2B findings, including (a) an incomplete MEN2B phenotype and (b) lack of RET gene mutations in the presence of MEN2B features. A few patients with multiple neuroma syndrome or MEN2B without MTC or a RET gene mutation have been described. ${ }^{3,10}$ There have been only a few other reports describing patients with MEN2B-like features. ${ }^{11-14}$ These cases, termed 'pure mucosal neuroma syndrome' by Gordon et al ${ }^{14}$ and by Spyer et $a l^{12}$, showed neither associated endocrinopathies nor RET mutations. The cases reported by Gómez et $a l^{11}$ and by Dennehy et $a l^{13}$ were familial, thus excluding mosaicism for MEN2 mutations as a possible explanation. There are more examples of phenotypic overlap suggesting the presence of additional, still undefined subgroups of disorders similar to MEN $2 \mathrm{~A}$ and 2B. For example, Kane et $a l^{15}$ reported a unique kindred manifesting with MTC and thickened corneal nerves, but without other clinical features of MEN2A or MEN2B and absence of $R E T$ gene mutations. Despite a number of described atypical cases, none included a developmental brain anomaly, non-mucosal nerve 
Table 1 Clinical findings in affected patients (including one similar case from the literature ${ }^{26}$ )

\begin{tabular}{|c|c|c|c|c|c|}
\hline & Patient 1 & Patient 2 & Patient 3 & Patient 4 & Meyer-Wobig case ${ }^{26}$ \\
\hline Bilateral multiple orbital neurofibromas & + & + & + & + & + \\
\hline Glaucoma & & & + & + & + \\
\hline Proptosis & + & + & + & + & + \\
\hline Full lips & + & + & + & + & + \\
\hline Mucosal neurofibromas & + & + & + & & + \\
\hline Peripheral neuropathy & & + & + & & \\
\hline Marfanoid habitus & + & + & + & + & + \\
\hline Pectus excavatum & + & & + & & + \\
\hline Scoliosis & & & + & & \\
\hline Neuronal migration anomaly & + & + & & & \\
\hline Keloids & + & + & & + & \\
\hline & SMARCB1 & SMARCB1 & & & \\
\hline & PTEN & PTEN & & & \\
\hline & $\mathrm{RET}^{\mathrm{a}}$ & PRKAR1A & & & \\
\hline & PRKAR1A & RET & & & \\
\hline & PTPN1 & $\mathrm{CGH}$ & & & \\
\hline & RAF1 & & & & \\
\hline & sos1 & & & & \\
\hline & KRAS & & & & \\
\hline & NRAS & & & & \\
\hline & $\mathrm{CBL}$ & & & & \\
\hline & SHOC1 & & & & \\
\hline & $\mathrm{CGH}^{\mathrm{b}}$ & & & & \\
\hline
\end{tabular}

neurofibromas, or 'neurofibromatous' neuropathy. Aside from a single description of a patient with bilateral, localized orbital neurofibromas and Charcot-Marie-Tooth disease, no other instances of a neuropathic process in patients with orbital neurofibromas or with MEN2B-like feature have been reported. ${ }^{16}$

Orbital neurofibromas are rare and usually sporadic. They are only infrequently (7-10\%) associated with NF1. ${ }^{17-21}$ Neurofibromas in the area of the eyelids and orbits often result in development of glaucoma $^{22,23}$ as was the case in two of our patients. Bilateral orbital involvement with neurofibromas is extremely unusual. Only a few instances, either as an isolated clinical finding ${ }^{16,24}$ or in association with multiple other features of NF1, have been reported. ${ }^{25}$ Meyer and Wobig $^{26}$ described a patient presenting with symmetric bilateral orbital neurofibromas and several features of MEN2B, including marfanoid habitus, enlarged corneal nerves, thickened lips and mucosal neuromas, but neither diagnostic testing for RET or NF1 mutations had been performed nor was information provided regarding associated features, such as neuropathy or a neuronal migration defect. ${ }^{26}$ Despite striking clinical similarities between cases reported by Meyer and Wobig ${ }^{26}$ and those of our patients, lack of additional information obviates the conclusion that they have had the same condition.

We propose that the patients reported herein represent a new syndrome characterized by bilateral orbital neurofibromas, painful peripheral neurofibromas, distinct facial features, marfanoid habitus, enlarged corneal nerves, and a neuronal migration defect. Additional feature of this new syndrome may be propensity for keloid scars and facial skin freckling including lips. We suggest that previously reported patients with 'pure mucosal neuroma syndrome' have a minor form of this condition. Its genetic basis remains to be determined.

\section{CONFLICT OF INTEREST}

The authors declare no conflict of interest.

\section{ACKNOWLEDGEMENTS}

This work was supported by the Fonds voor Wetenschappelijk Onderzoek (FWO)-Vlaanderen (G.0578.06) (EL), a Concerted Action Grant (GOA/11/010) from the KULeuven, the FWO scientific research community (W0.027.09) (EL) and the Interuniversity Attraction Poles (IAP) granted by the Federal Office for 
Scientific, Technical and Cultural Affairs, Belgium (2007-2011; P5/25) (EL). HB was supported by the Institute for the Promotion of Innovation through Science and Technology in Flanders (IWT-Vlaanderen). Additional support was provided by NF Inc., MN, USA (NFIM\#2) (DBV). We thank Kara A Mensink, MS, CGC, for helpful edits and Cheryl Dowse for technical assistance.

1 Maertens O, De Schepper S, Vandesompele J et al: Molecular dissection of isolated disease features in mosaic neurofibromatosis type 1. Am J Hum Genet 2007: 81: 243-251.

2 Carney JA: Carney complex: the complex of myxomas, spotty pigmentation, endocrine overactivity, and schwannomas. Semin Dermatol 1995; 14: 90-98.

3 Morrison PJ, Nevin NC: Multiple endocrine neoplasia type 2B (mucosal neuroma syndrome, Wagenmann-Froboese syndrome). J Med Genet 1996; 33: 779-782.

4 Schaffer JV, Kamino H, Witkiewicz A, McNiff JM, Orlow SJ: Mucocutaneous neuromas: an underrecognized manifestation of PTEN hamartoma-tumor syndrome. Arch Dermatol 2006; 142: 625-632.

5 Hartlapp I, Buhring U, Dichgans J, Isenmann S: A patient with Marfan's syndrome and neurofibromatosis type 1 with polyneuropathy. Eur J Neurol 2004; 11: 641-644.

6 Sommer C, Kress M: Recent findings on how proinflammatory cytokines cause pain: peripheral mechanisms in inflammatory and neuropathic hyperalgesia. Neuroscience Letters 2004; 361: 184-187.

7 Watson JJ, Allen SJ, Dawbarn D: Targeting nerve growth factor in pain: what is the therapeutic potential? BioDrugs 2008; 22: 349-359.

8 Elisei R, Cosci B, Romei C et al: RET exon 11 (G691S) polymorphism is significantly more frequent in sporadic medullary thyroid carcinoma than in the general population. J Clin Endocrinol Metab 2004; 89: 3579-3584.

9 Robledo M, Gil L, Pollán M et al: Polymorphisms G691S/S904S of RET as genetic modifiers of MEN 2A. Cancer Res 2003; 63: 1814-1817.

10 Parker DG, Robinson BG, O'Donnell BA: External ophthalmic findings in multiple endocrine neoplasia type 2B. Clin Exp Ophthalmol 2004; 32: 420-423.

11 Gómez JM, Biarnés J, Volpini V, Martí T: Neuromas and prominent corneal nerves without MEN 2B. Ann Endocrinol (Paris) 1998; 59: 492-494.
12 Spyer G, Ellard S, Turnpenny PD, Hattersley AT, Vaidya B: Phenotypic multiple endocrine neoplasia type $2 \mathrm{~B}$, without endocrinopathy or RET gene mutation: implications for management. Thyroid 2006; 16: 605-608.

13 Dennehy PJ, Feldman GL, Kambouris M, O'Malley ER, Sanders CY, Jackson CE: Relationship of familial prominent corneal nerves and lesions of the tongue resembling neuromas to multiple endocrine neoplasia type 2B. Am J Ophthalmol 1995; 120: 456-461.

14 Gordon CM, Majzoub JA, Marsh DJ et al: Four cases of mucosal neuroma syndrome: multiple endocrine neoplasm 2B or not 2B? J Clin Endocrinol Metab 1998; 83: 17-20.

15 Kane LA, Tsai MS, Gharib $\mathrm{H}$ et al: Familial medullary thyroid cancer and prominent corneal nerves: clinical and genetic analysis. J Clin Endocrinol Metab 1995; 80: 289-293.

16 Ma'luf RN, Noureddin BN, Ghazi NG, Tawil AN, Allam SS: Bilateral, localized orbital neurofibromas and Charcot-Marie-Tooth disease. Arch Ophthalmol 2005; 123: $1443-1445$.

17 Jackson IT, Laws ERJ, Martin RD: The surgical management of orbital neurofibromatosis. Plast Reconstr Surg 1983; 71: 751-758.

18 Shields JA, Shields CL, Lieb WE, Eagle RC: Multiple orbital neurofibromas unassociated with von Recklinghausen's disease. Arch Ophthalmol 1990; 108: 80-83.

19 Krohel GB, Rosenberg PN, Wright JE, Smith RS: Localized orbital neurofibromas. Am J Ophthalmol 1985; 100: 458-464.

20 Rootman J, Robertson WD: Neurogenic tumors. In: Rootman J (ed.). Diseases of the Orbit. Philadelphia: JB Lippincott, 1988, pp 281-334.

21 Gurland JE, Tenner M, Hornblass A, Wolintz AH: Orbital neurofibromatosis: involvement of the orbital floor. Arch Ophthalmol 1976; 94: 1723-1725.

22 Morales J, Chaudhry IA, Bosley TM: Glaucoma and globe enlargement associated with neurofibromatosis type 1. Ophthalmology 2009; 116: 1725-1730.

23 Colas-Tomas T, Gutierrez-Diaz E, Tejada-Palacios P, Barcelo-Mendiguchia A, MenciaGutierrez E: Management of congenital glaucoma in neurofibromatosis type 1: a report of two cases. Int Ophthalmol 2010; 30: 211-214.

24 Earley MJ, Moriarty P, Yap LH: Isolated bilateral orbital neurofibromatosis in a twelve-year-old. Br J Plast Surg 2001; 54: 162-164.

25 Bloem JJ, van der Meulen JC: Neurofibromatosis in plastic surgery. Br J Plast Surg 1978; 31: 50-53.

26 Meyer DR, Wobig JL: Bilateral localized orbital neurofibromas. Ophthalmol 1992; 99 : 1313-1317. 